\title{
Avaliação Comparativa entre os Nanocompósitos de Argila Motmorilonita/LLDPE e com Hexaniobato de Potássio/LLDPE: Caracterização das Propriedades Mecânicas e de Transporte
}

\author{
Daniel Komatsu, Harumi Otaguro \\ Departamento de Química, UFSCar \\ Adhemar C. Ruvolo Filho \\ Departamento de Engenharia de Materiais, UFSCar
}

\begin{abstract}
Resumo: Nanocompósitos de LLDPE/argila montmorilonita e LLDPE/niobato organofilizado foram obtidos através da diluição de um concentrado da respectiva carga em uma extrusora dupla rosca obtendo-se concentrações finais de 1,5 a 10,0\% em carga. No presente estudo utilizou-se o ensaio mecânico de tração e o de permeação ao vapor de água e ao oxigênio para caracterizar estes nanocompósitos. No ensaio mecânico de tração observou-se aumento no valor do módulo elástico e diminuição no valor da tenacidade com o aumento da concentração da argila montmorilonita, o que era esperado devido à rigidez da carga. Comportamento semelhante também foi observado no caso dos nanocompósitos de niobato organofilizado, porém de maneira menos acentuada devido à estrutura química do niobato de potássio. Com auxílio do MEV/FEG observou-se que a distribuição da argila é superior a do niobato organofilizado para concentrações inferiores a 10,0\% de carga. No ensaio de permeação ocorre diminuição no valor de permeabilidade com o aumento da concentração da argila montmorilonita para ambos os gases utilizados. Na presença do niobato organofilizado observa-se diminuição, seguido de aumento no valor da permeação com o aumento da concentração da carga para ambos os gases utilizados. Além disso, observou-se que a polaridade do gás utilizado é um fator importante no processo de difusão através do nanocompósito.
\end{abstract}

Palavras-chave: Nanocompósito, polietileno linear de baixa densidade, argila montmorilonita, hexaniobato de potássio, propriedade mecânica e propriedade de transporte de gás.

\section{Comparative Evaluation between Montmorillonite Clay/LLDPE and Potassium Hexaniobate/LLDPE Nanocomposites: Characterization of Mechanical and Transport Properties}

Abstract: Linear low density polyethylene-montmorillonite clay and linear low density polyethylene-organophilic
niobate nanocomposites were obtained from dilution of masterbatch with $20 \% \mathrm{w} / \mathrm{w}$ of fillers in the LLDPE matrix
by melt intercalation using a twin-screw extruder, obtaining nanocomposites with $1.5 \%$ up to $10.0 \%$ w/w of filler. In
this study mechanical and water vapor and oxygen permeation tests were used to characterize the nanocomposites.
In mechanical tests an increase of modulus values and decrease of toughness value by increasing concentration of
montmorillonite clay were observed. The behavior of LLDPE-organophilic niobate nanocomposites was similar
to LLDPE-montmorillonite clay nanocomposites but softer due to hexaniobate structure. The distribution of the
organoclay is more homogeneous than organophilic niobate to concentrations below $10.0 \%$ filler using the SEM/
FEG. It is possible to see a decrease in the permeability value with increasing concentration of montmorillonite clay
for both gases used. In the LLDPE-organophilic niobate nanocomposites a decrease of permeability value occurs
followed by an increase of permeability value for both gases used, with increasing concentration of organophilic
niobate. Furthermore, it was observed that the polarity of the gas used is an important factor in the diffusion process
through the nanocomposite.

Keywords: Nanocomposite, linear low density polyethylene, montmorillonite clay, potassium hexaniobate, mechanical property and transport property.

\section{Introdução}

O Polietileno é uma das poliolefinas mais consumidas no mundo, sendo altamente utilizada devido a sua versatilidade em termos de propriedade, aplicação e principalmente ao baixo custo. Nos últimos anos, ele

tem sido usado em aplicações para embalagens ${ }^{[1]}$ e por isso é de extrema importância o entendimento de sua morfologia, pois é um ponto importante na explicação do processo de transporte. Em função da permeabilidade 
das poliolefinas a gases $\left(\mathrm{O}_{2}\right.$ e vapor de água) tem-se buscado melhorias nas propriedades de barreira com a incorporação de cargas tradicionais como é o caso da sílica, fibra de vidro, talco, etc., formando os microcompósitos tradicionais. O transporte de pequenas moléculas através das membranas poliméricas ocorre devido ao movimento aleatório das moléculas individuais. Dessa maneira o processo de transporte de gás pelo polímero, ocorre através de um fenômeno cujo mecanismo inicial deve-se a sorção superficial das moléculas na superfície do polímero, seguido pela condensação dessas moléculas no interior do polímero e posteriormente pelo processo de difusão das mesmas. A equação que define termodinamicamente esse processo é descrita em termos dos processos de sorção e de difusão da seguinte maneira: $\mathrm{P}=\mathrm{S}$.D, onde o termo $\mathrm{S}$ é de natureza termodinâmica, sendo determinado pela condensabilidade inerente do gás através das interações polímero/gás e pelo excesso de volume livre existente no polímero. O termo $\mathrm{D}$ é de natureza cinética e reflete a mobilidade do gás na matriz polimérica, ou seja, está relacionado ao processo de difusão ${ }^{[2]}$. Porém existem alguns fatores que influenciam esse processo. No caso da solubilidade (S) ela é afetada com a facilidade de condensação do gás no interior do polímero, a presença de volume livre ${ }^{[3]} \mathrm{e}$ a polaridade relativa do trio gás/matriz polimérica/carga. O processo de difusão (D), por sua vez, é afetado, também, pela presença de volume livre, pelo grau de cristalinidade da matriz polimérica, uma vez que a difusão ocorre exclusivamente na fase amorfa do polímero ${ }^{[4]}$. Além disso, a difusão também é afetada pela tortuosidade ${ }^{[5,6]}$ na matriz polimérica, gerado nesse caso pela presença de cargas inorgânicas que, de acordo com a maneira que está distribuída e dispersa, aumenta o caminho livre médio para a difusão.

Nas últimas décadas houve grande interesse, tanto das universidades, quanto das indústrias, no chamado nanocompósito. Ele é denominado um material híbrido, composto por uma matriz polimérica na qual se encontra dispersa uma fase inorgânica constituída de partículas com elevada razão de aspecto e dimensões tipicamente nanométricas ${ }^{[7,8]}$. A vantagem da utilização de nanocarga em relação à carga tradicional é a melhoria gerada nas propriedades mecânica, térmica e de barreira do nanocompósito com a adição de uma pequena quantidade de carga e, também, por não ser necessário nenhum ajuste nos equipamentos utilizados para o processamento do $\operatorname{mesmo}^{[9-11]}$.

Os argilo-minerais, assim como os sólidos inorgânicos lamelares apresentam estrutura formada pela sobreposição organizada de camadas bidimensionais chamadas de lamelas. Entre essas camadas, existe um espaço livre denominado de região interlamelar. Esse espaço pode estar vazio, no caso do composto ser formado por lamelas eletricamente neutras ou estar preenchido por íons que mantém a eletroneutralidade do sistema, no caso da lamela apresentar excesso de cargas elétricas. $\mathrm{O}$ interesse em compostos com esse tipo de estrutura reside no fato de que a região interlamelar pode ser modificada de modo a abrigar espécies de diferentes naturezas, conferindo novas propriedades ao sistema a partir da interação de natureza elétrica que se estabelece entre as partes. $\mathrm{O}$ hexaniobato de fórmula $\mathrm{K}_{4} \mathrm{Nb}_{6} \mathrm{O}_{17}$ está incluído na categoria destes materiais, o qual é formado por camadas constituídas de unidades octaédricas distorcidas de $\mathrm{NbO}_{6}$, que estão ligadas entre si pelo compartilhamento de vértices e arestas. A região interlamelar é preenchida por íons de metais alcalinos $\left(\mathrm{K}^{+}, \mathrm{Rb}^{+}, \mathrm{Cs}^{+}\right)$mantendo a eletroneutralidade do sistema. Esse sólido apresenta duas regiões interlamelares distintas designadas por regiões I e II, como pode ser visto na Figura 1b. Essa diferenciação é feita não só em razão das regiões serem cristalograficamente diferentes, mas, também, por apresentarem propriedades distintas relacionadas com a intercalação de moléculas. Dos estudos realizados por Gasperin e Bihan ${ }^{[12]}$ sobre mecanismos de hidratação da região interlamelar, conclui-se que apenas a região I pode ser hidratada. Essa diferença de comportamento entre as duas regiões faz com que a região I seja muito mais reativa frente à substituição dos cátions interlamelares por reações de troca iônica do que a região II.

Atualmente de forma quase generalizada, os estudos envolvendo o desenvolvimento de novos materiais nano estruturados em matriz polimérica, tem feito uso dos argilo-minerais da classe das montmorilonitas. Embora

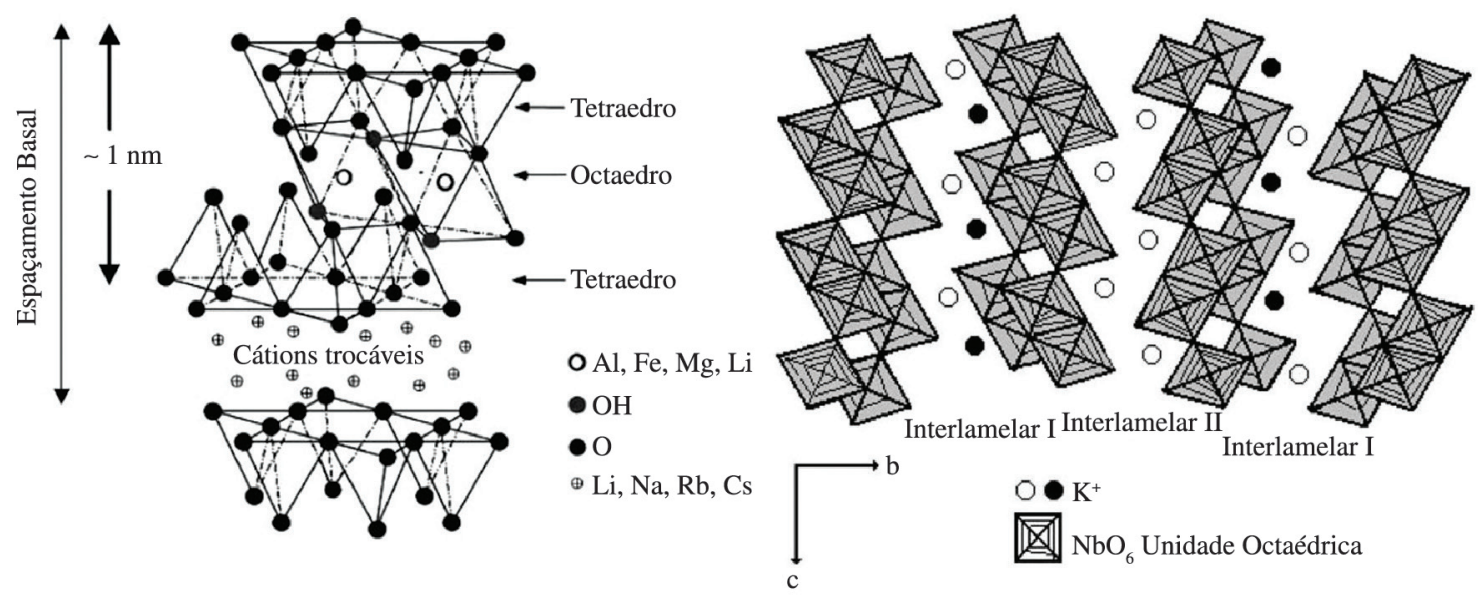

Figura 1. Representação esquemática da estrutura lamelar da argila montmorilonita (Figura 1a) ${ }^{[13]}$ e da estrutura lamelar do hexaniobato de potássio (Figura 1b) ${ }^{[14]}$. 
seja um material de origem natural, o maior problema na sua utilização reside no grau de pureza, o que reduz muito a disponibilidade de aquisição destes materiais em muitos países, incluindo o Brasil. Além disso, as argilas brasileiras apresentam, mesmo após os procedimentos de purificação, teores de íons ferro suficientemente elevados (em geral da ordem de $2-3 \%$ ), sendo este um fator inconveniente, pois esse metal pode atuar como catalisador de processos termo-oxidativos da matriz polimérica olefínica durante as etapas de processamento do compósito no estado fundido. Por outro lado, a obtenção de sólidos inorgânicos lamelares via rotas sintéticas apresenta-se como uma alternativa atraente, uma vez que os produtos obtidos têm grau de pureza e homogeneidade de tamanho de partículas, a nível atômico, extremamente elevado ${ }^{[15]}$.

No presente estudo foi utilizada como nanocargaa argila montmorilonita que é formada por estrutura cristalina de silicatos em camadas de $2: 1$, a qual consiste de camadas bidimensionais onde uma folha central octaédrica de alumina é fundida a dois tetraedros de sílica externo pela ponta, conforme Figura $1 \mathrm{a}^{[13,16]}$. O hexaniobato de potássio é constituído por tetraedros distorcidos, podendo ligar-se tanto pelos vértices, quanto pelas arestas (Figura 1b) ${ }^{[14,17]}$, o qual foi sintetizado em nosso laboratório, apresentando ao final uma estrutura na forma de empilhados lamelares semelhantes à estrutura da argila montmorilonita.

Portanto, o objetivo deste trabalho é investigar a influência de diferentes cargas manométricas, tendo como base a mesma matriz polimérica (LLDPE), na propriedade mecânica e de transporte destes nanocompósitos. Ou seja, avaliar de forma comparativa o niobato organofilizado e a argila montmorilonita organofilizada comercial Cloisite 20A. O estudo envolvendo o niobato organofilizado como nanocarga é uma rota alternativa às nanocargas tradicionais como no caso da argila montmorilonita Cloisite 20A, uma vez que esta argila é uma matériaprima importada. Apesar das argilas nacionais serem encontradas em abundância, as mesmas apresentam altos teores de íons ferro os quais agem como catalisadores na reação de degradação termo-oxidativa nos polímeros, em geral na etapa de processamento por extrusão.

Para este intuito foram utilizadas as seguintes análises: ensaio mecânico de tração, microscopia eletrônica de varredura de alta resolução (MEV/FEG) e ensaio de permeação com gases que apresentam diferentes polaridades $\left(\mathrm{O}_{2} \text { e vapor de água }\right)^{[18,19]}$.

\section{Materiais e Métodos}

Os materiais utilizados neste trabalho foram: Polietileno Linear de Baixa Densidade (LLDPE, MFI=0,5 e $1,5 \mathrm{~g} / 10 \mathrm{~min}$ ) LF-0720/21AF da Braskem; argila montmorilonita organofílica (Southern Clay, Cloisite 20A) modificada com sal de amônio quaternário com dois radicais alquila de cadeia longa entre 14 e 18 átomos de carbono; hexaniobato de potássio que foi obtido a partir do aquecimento do óxido de nióbio com o carbonato de potássio a $1100^{\circ} \mathrm{C}$ por um período de 10 horas. Após esse período o sólido obtido foi triturado e colocado em um balão contendo ácido nítrico a $60^{\circ} \mathrm{C}$ durante um período de 3 dias. Nesta etapa ocorrerá a formação do niobato protônico $\left(\mathrm{H}_{2} \mathrm{~K}_{2} \mathrm{Nb}_{6} \mathrm{O}_{17}\right)$ a partir de uma reação de troca iônica do composto $\mathrm{K}_{4} \mathrm{Nb}_{6} \mathrm{O}_{17}$ em meio ácido. A reação de troca iônica é importante, pois o produto formado irá favorecer a obtenção do niobato organofilizado intercalado. A intercalação da octadecilamina (Aldrich ${ }^{\circledR}$ ) foi realizada via reação ácido-base entre o niobato na forma protônica $\left(\mathrm{H}_{2} \mathrm{~K}_{2} \mathrm{Nb}_{6} \mathrm{O}_{17}\right)$ e a amina em uma mistura de solventes etanol/água $(75 / 25, \mathrm{v} / \mathrm{v})$ por $24 \mathrm{~h}$ a $60^{\circ} \mathrm{C}^{[20]}$; Polietileno Linear de Baixa Densidade enxertado com alto teor de anidrido maléico de acordo com a informação do fabricante (LLDPE-g-MA, DuPont, Fusabond MX110D, MFI=22,9 g/10 min) fornecido pela empresa Cromex.

Foi preparado, inicialmente, um concentrado da argila e do hexaniobato de potássio separadamente, misturando-se cada tipo de carga ao LLDPE-g-MA na proporção de 1:2 em um misturador tipo Drais, $(\mathrm{w}=3000$ rpm) produzindo ao final um concentrado com $20,0 \%$ em massa de nanocarga. Em seguida o concentrado foi moído criogenicamente em um moinho modelo IKA Werke M20 com rotação de $20000 \mathrm{rpm}$, sendo posteriormente diluído no LLDPE para obtenção dos nanocompósitos via intercalação no estado fundido, utilizando-se uma extrusora dupla rosca modelo MT19TC-25:1 da B \& P Process Equipament and Systems, $\mathrm{L} / \mathrm{D}=25$, com o seguinte perfil de temperatura: $150^{\circ} \mathrm{C} / 170^{\circ} \mathrm{C} / 170^{\circ} \mathrm{C} / 200^{\circ} \mathrm{C} / 200^{\circ} \mathrm{C}$ e velocidade de rotação das roscas de $120 \mathrm{rpm}$. Dessa maneira, foram obtidos nanocompósitos com concentrações que variaram de $1,5 \%$ até $10,0 \%$ em massa. Placas dos respectivos nanocompósitos foram obtidas via termoprensagem na temperatura de $180^{\circ} \mathrm{C}$, seguido de resfriamento sob pressão até a temperatura ambiente. $\mathrm{O}$ ensaio mecânico foi realizado em um equipamento universal de ensaio mecânico marca EMIC DL 2000, na temperatura ambiente $\left(25^{\circ} \mathrm{C}\right)$ e com velocidade de estiramento de $10 \mathrm{~mm} / \mathrm{min}$, sendo o ensaio feito em quadruplicata. Os corpos de prova tipo 2 foram confeccionados segundo a norma ASTM D638. O ensaio de permeação ao oxigênio foi realizado no equipamento Oxitran ${ }^{\circledR}$ da Mocon que possui duas câmaras de permeação. No teste foram utilizados dois filmes com espessura média de $100 \mu \mathrm{m}$, os quais foram colocados separadamente nos respectivos compartimentos de medição da célula de permeação. Os filmes poliméricos a serem testados foram recobertos por uma máscara de alumínio dos dois lados, ficando com uma área exposta de $5 \mathrm{~cm}^{2}$ para o teste. Inicialmente o equipamento ficou em condicionamento por uma hora, com um fluxo de gás nitrogênio, para garantir que a câmara onde está o detector não tenha presença de gás oxigênio e também, para aclimatar a amostra afim que a mesma alcance o equilíbrio. Após esse tempo de condicionamento o ensaio de permeação se inicia e o gás de teste (gás oxigênio) entra em contato com um lado do filme polimérico, sendo permeado para o outro lado do filme e carregado por um fluxo de gás nitrogênio, gerando uma mistura desses gases. Essa mistura de gases passa através do sensor e dessa maneira a quantidade de oxigênio contida no gás de transporte é medida, determinando-se, assim, a taxa de transmissão do gás oxigênio através do filme polimérico. A medida 
é tomada em 6 medições com um tempo individual de 15 minutos cada, totalizando um tempo total de ensaio de aproximadamente 1,5 horas para cada célula. O ensaio é baseado nas normas ASTM D-3985, ASTM F-1927, JIS K-7126, ASTM F-1307 e ISO CD 15105-2. As análises foram realizadas à temperatura ambiente e em duplicata. O ensaio de permeação ao vapor de água foi feito pelo sistema de pesagem utilizando-se um copo de Payne no interior do qual, é colocada água destilada. O fechamento do copo se dá através de um sistema de flange onde é colocado o filme polimérico entre dois anéis de borracha. O conjunto é, então, colocado dentro de um dessecador na presença de um agente dessecante, que nesse caso foi utilizado o pentóxido de fósforo (com a finalidade de gerar gradiente de umidade relativa entre o interior do copo e o interior do dessecador). Desta forma, este gradiente de pressão gerado dentro do dessecador permite que ocorra a permeação do vapor de água através do filme polimérico. Inicialmente os copos de Payne são pesados em curtos intervalos de tempo, passando, posteriormente, a intervalos maiores à medida que a variação de massa seja pequena. Essa variação é diretamente proporcional à massa de água que passa através do filme polimérico. $\mathrm{O}$ experimento é feito dentro de um banho ajustado na temperatura ambiente e em triplicata. A microscopia eletrônica de varredura de alta resolução (MEV/FEG) foi feita em um microscópio eletrônico de alta resolução (FEG) modelo supra 35-Zeiss na superfície fraturada criogenicamente do corpo de prova.

\section{Resultados e Discussão}

A Figura 2 ilustra os gráficos do balanço entre módulo elástico versus tenacidade em função da porcentagem de carga para os nanocompósitos preenchidos com argila montmorilonita e com niobato organofilizado.

Observa-se na Figura 2 a que a presença da argila montmorilonita eleva o valor do módulo elástico da maioria dos nanocompósitos comparado ao LLDPE puro e extrudado, exceto para concentração de $5,0 \%$. No entanto considerando-se a barra de erro o valor é praticamente o mesmo da amostra pura. Os nanocompósitos com 7,5

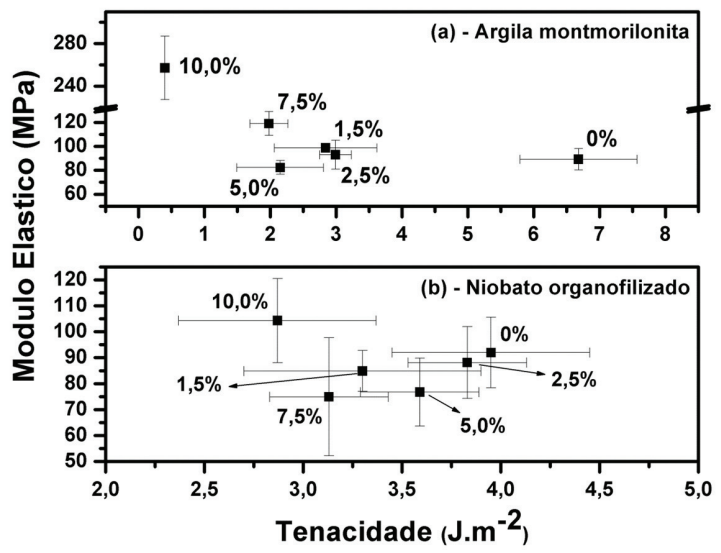

Figura 2. Gráfico do balanço entre Módulo Elástico Vs Tenacidade em função da porcentagem de carga (nanocompósitos com argila montmorilonita (Figura 2a) e com niobato organofilizado (Figura $2 b$ )). e $10,0 \%$ de argila foram os que apresentaram aumentos mais acentuados, com ganho próximo a 33\% e 188\%, respectivamente. Esse aumento é devido à formação de grandes aglomerados de argila na matriz do LLDPE, os quais se sobrepõem ao tamanho das cadeias do polímero e com isso, durante o ensaio mecânico de tração o esforço é concentrado sobre os tactóides e não na matriz polimérica. Dessa maneira, como o valor do módulo elástico da argila $(178 \mathrm{GPa})^{[21]}$ é muito superior ao do polímero puro, então, o valor do módulo elástico desses nanocompósitos aumentam muito. Para os demais nanocompósitos (1,5; 2,5 e $5,0 \%$ ) estes aumentos devem-se a boa adesão interfacial polímero/argila, que é resultante da boa distribuição da argila na matriz polimérica, fato que será corroborado com os resultados de MEV.

Os nanocompósitos preenchidos com niobato organofilizado estão ilustrados na Figura 2b. Nesse caso observa-se uma queda nos valores dos módulos elásticos para a maioria dos nanocompósitos em relação ao LLDPE extrudado, exceto para concentração de 10,0\% de niobato organofilizado que apresentou aumento de aproximadamente $13,4 \%$ e redução no valor de tenacidade. Comparando a diferença nos valores dos módulos elásticos entre os nanocompósitos preenchidos com $7,5 \%$ de argila montmorilonita e com o de $10,0 \%$ de niobato organofilizado, em relação ao polímero puro, observa-se que o valor do módulo elástico no primeiro caso é praticamente 2,5 vezes maior do que no segundo caso. Essa diferença nos valores dos módulos elásticos dos nanocompósitos está relacionada com as diferentes estruturas lamelares apresentadas pelas nanocargas. No caso da argila montmorilonita a sua estrutura é formada por um octaedro fundido a dois tetraedros pela ponta e uma região interlamelar. $\mathrm{O}$ niobato organofilizado por sua vez é formado de tetraedros distorcidos, podendo se ligar tanto pelo vértice, quanto pelas arestas e, além disso, apresentam dois tipos de regiões interlamelares cristalograficamente diferentes, sendo que a modificação orgânica ocorre em apenas uma delas ${ }^{[14]}$. Além disso, é observada uma diminuição nos valores de tenacidade para as amostras preenchidas tanto com argila montmorilonita, quanto com niobato organofilizado. Isso mostra que ambas as cargas deixam os nanocompósitos mais rígidos do que o LLDPE puro, porém a argila montmorilonita é a que proporciona a maior redução.

As Figuras 3 e 4 ilustram as micrografias eletrônicas de varredura da superfície fraturada criogenicamente dos nanocompósitos de LLDPE preenchidos com argila montmorilonita e niobato organofilizado, respectivamente.

Nota-se que tanto a argila $(1,5$ e $5,0 \%)$ quanto o niobato organofilizado $(1,5 \%)$ apresentaram boa distribuição pela matriz de LLDPE em baixas concentrações de nanocarga. Por outro lado, nos nanocompósitos com argila montmorilonita e com elevada concentração de carga (7,5 e 10,0\%) essa distribuição é ligeiramente prejudicada. Já no caso dos nanocompósitos com niobato organofilizado e com elevada concentração de carga (7,5 e 10,0\%) há piora na distribuição da mesma pela matriz polimérica. Além disso, comparando as duas cargas observa-se que a argila apresenta uma melhor 


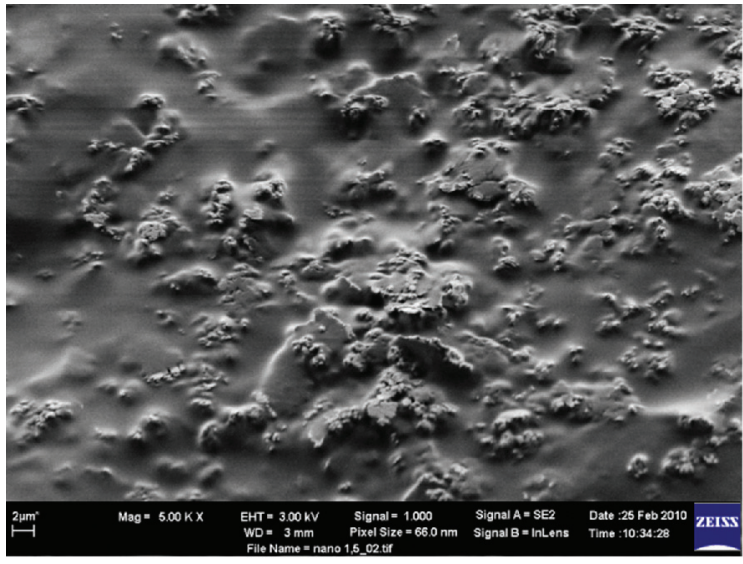

(a)

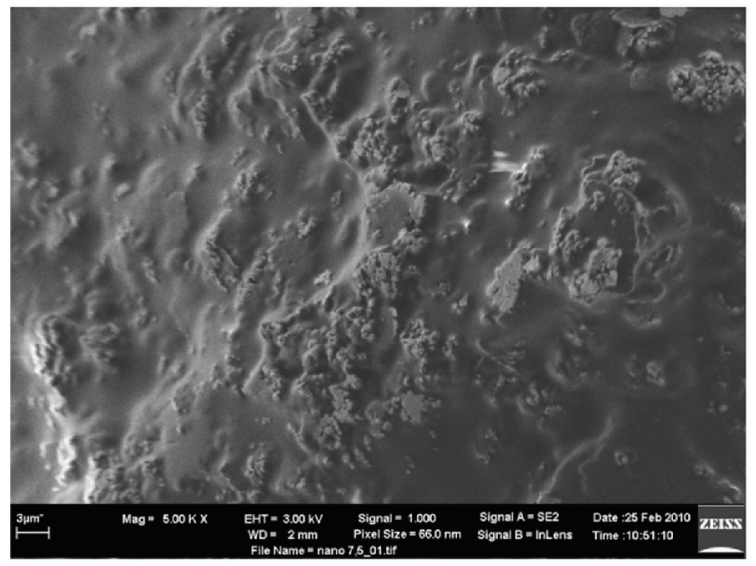

(c)

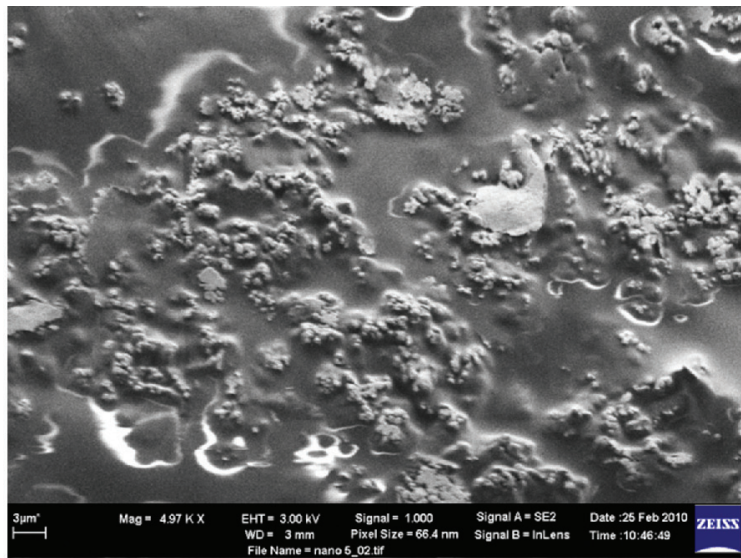

(b)

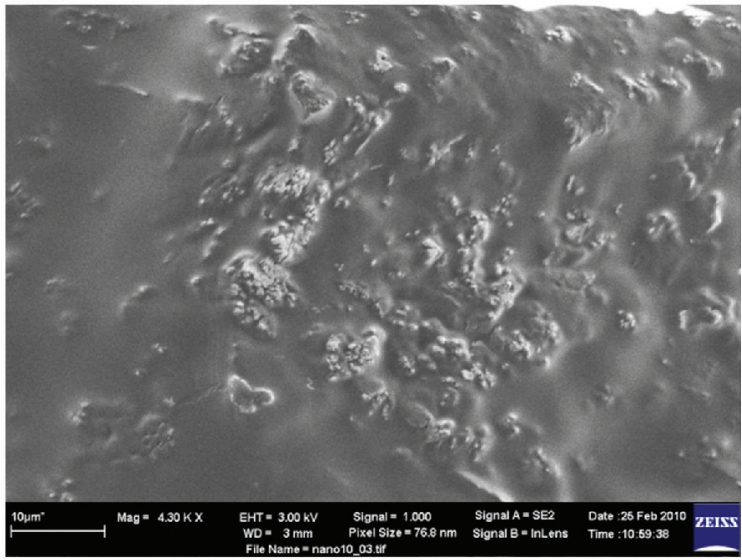

(d)

Figura 3. Micrografias dos nanocompósitos com (a)1,5\%; (b)5,0\%; (c)7,5\% e (d)10,0\% de argila montmorilonita. A barra dimensional nas Figuras 3(a), 3(b), 3(c) e 3(d) valem $2 \mu \mathrm{m}, 3 \mu \mathrm{m}, 3 \mu \mathrm{m}$ e $10 \mu \mathrm{m}$, respectivamente.

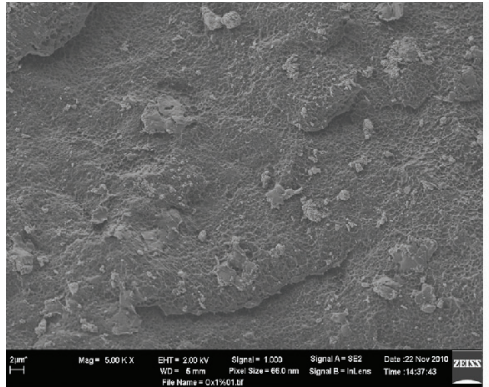

(a)

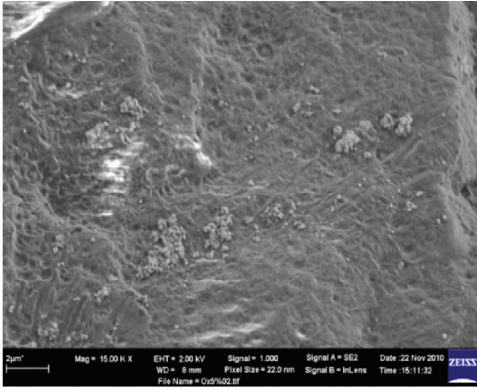

(b)

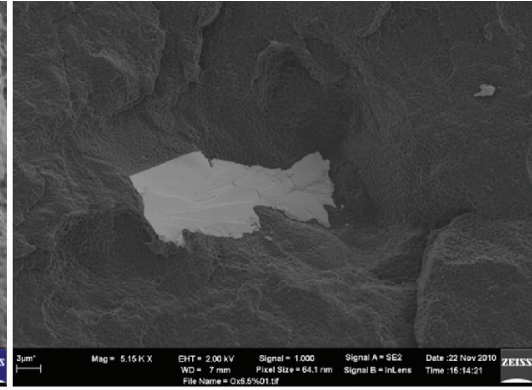

(c)

Figura 4. Micrografias dos nanocompósitos com (a)-1,5\%; (b)-7,5\% e (c)-10,0\% de óxido lamelar. A barra dimensional nas Figuras 4(a), 4(b) e 4(c) valem $2 \mu \mathrm{m}, 2 \mu \mathrm{m}$ e $3 \mu \mathrm{m}$, respectivamente.

distribuição pela matriz do LLDPE do que o niobato organofilizado. Portanto, pode-se dizer que o tipo de carga e a concentração da mesma é um fator que limita sua distribuição no LLDPE, e conseqüentemente influencia sua propriedade mecânica como discutido anteriormente.

Além disso, um aspecto importante a ser mencionado está relacionado ao método de processamento utilizado na obtenção dos nanocompósitos, o qual gera agregados com diversos tamanhos. No caso dos nanocompósitos com argila montmorilonita o tamanho desses agregados vai desde $1 \mu \mathrm{m}$ para os nanocompósitos com 1,5; 5,0 e $7,5 \%$ até $10 \mu \mathrm{m}$ para o nanocompósito com $10,0 \%$.

Por sua vez, os nanocompósitos com niobato organofilizado apresentaram agregados com tamanho que vai desde $1 \mu \mathrm{m}$ para os nanocompósitos com 1,5 e $7,5 \%$ até $15 \mu \mathrm{m}$ para o nanocompósito com $10,0 \%$ (placa com cor mais clara). A partir desses valores de tamanho dos agregados é possível notar que nesse caso, 
independentemente de se utilizar argila montmorilonita ou niobato organofilizado, os agregados apresentaram tamanhos próximos, indicando que a diferença na estrutura lamelar das nanocargas não interfere no tamanho final dos agregados.

A Figura 5 apresenta o gráfico comparativo da permeabilidade relativa entre oxigênio e vapor de água em função da porcentagem de argila montmorilonita (5a) e de niobato organofilizado (5b).

Observando os resultados de permeação que são apresentados na Figura 5a e na Tabela 1, nanocompósito preenchido com argila montmorilonita, é possível perceber que os valores de permeabilidade para o oxigênio diminuem de forma gradativa com o aumento da concentração de argila. Na presença de vapor de água e com aumento no teor de argila nota-se um acentuado crescimento no valor da permeabilidade para a concentração de 1,5\%, seguido de uma queda desses valores em concentrações maiores.

É interessante notar que os resultados obtidos através do ensaio mecânico de tração e as micrografias dos nanocompósitos preenchidos com argila montmorilonita ajudam a explicar a diminuição no valor da permeabilidade ao gás. No caso a boa adesão interfacial entre polímero/ argila montmorilonita e a boa distribuição da mesma pela matriz do LLDPE nos nanocompósitos com 1,5; 2,5 e $5,0 \%$ de argila observada no ensaio mecânico e

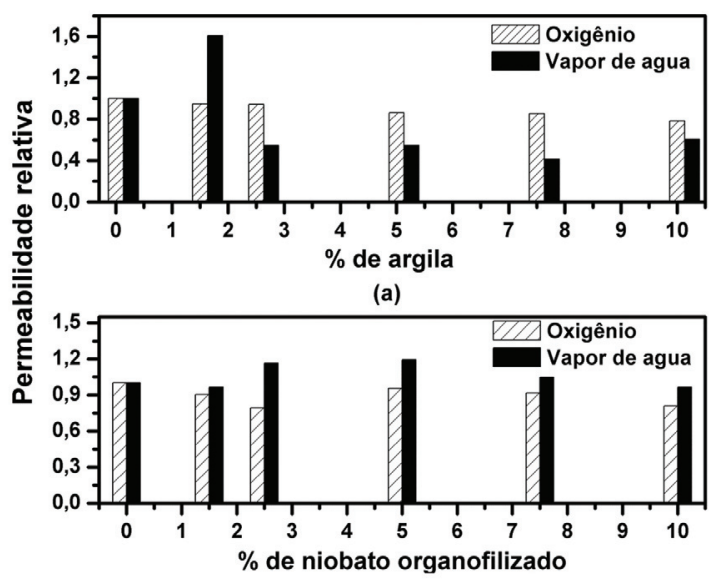

(b)

Figura 5. Gráfico comparativo da permeabilidade relativa entre $\mathrm{O}_{2}$ e vapor de água em função da porcentagem de argila montmorilonita (Figura 5a) e de niobato organofilizado (Figura 5b). nas micrografias, respectivamente, são responsáveis pelo aumento da tortuosidade. Com isso, o caminho que o gás irá percorrer dentro dessas amostras será aumentado, levando a uma queda no valor da permeabilidade nos nanocompósitos em relação ao LLDPE extrudado ${ }^{[22,23]}$. Apesar das amostras com maior concentração de argila (7,5 e 10,0\%) apresentar uma piora na distribuição da mesma pela matriz do LLDPE, como visto nas micrografias, a presença dos grandes agregados aumenta a tortuosidade na matriz do LLDPE e assim, reduz, também, a permeabilidade nessas amostras.

No caso dos resultados apresentados na Figura $5 \mathrm{~b}$ e na Tabela 1 observa-se um comportamento semelhante para os dois gases testados. Ou seja, há inicialmente uma tendência na diminuição dos valores de permeabilidade, seguido de um aumento e posterior tendência ao decaimento desses valores à medida que o teor de niobato organofilizado na amostra aumenta. Porém cabe ressaltar que estatisticamente, a tendência à redução nos valores da permeabilidade somente tem significado para o caso do oxigênio e até a concentração de 3,0\% do niobato organofilizado. Esse comportamento diferente dos nanocompósitos com niobato organofilizado em relação aos nanocompósitos com argila montmorilonita, poderia ser explicado, como no caso anterior, pelos resultados do ensaio mecânico de tração e das micrografias. Dessa maneira, essa diferença no comportamento de barreira poderia ser atribuída à menor adesão interfacial polímero/ niobato organofilizado, pois de acordo com o observado no ensaio mecânico de tração esses nanocompósitos não apresentaram aumento significativo no valor do módulo elástico em relação ao LLDPE extrudado, diferentemente dos nanocompósitos com argila montmorilonita. Isso seria uma das possíveis justificativas para o não melhoramento na propriedade de barreira, mesmo após a adição do niobato organofilizado.

A Tabela 1 ilustra os valores absolutos em Barrer da permeação de vapor de água e de oxigênio para os nanocompósitos com argila montmorilonita e com niobato organofilizado, juntamente com seus respectivos valores de desvios (d).

Observando-se os dados ilustrados na Tabela 1, nota-se que os valores de permeabilidade ao vapor de água são menores do que ao oxigênio, mesmo sendo o seu volume molecular maior (volume da molécula de $\mathrm{H}_{2} \mathrm{O}\left(3,68.10^{-30} \mathrm{~m}^{3}\right)$ do que o volume da molécula de $\left.\mathrm{O}_{2}\left(0,9 \cdot 10^{-30} \mathrm{~m}^{3}\right)\right)$. Dessa maneira, pode-se dizer que nesse caso os nanocompósitos são mais seletivos ao vapor de água do que ao oxigênio. Uma possível

Tabela 1. Valores de permeação dos gases $\mathrm{O}_{2}$ e vapor de $\mathrm{H}_{2} \mathrm{O}$ apresentados em Barrer*.

\begin{tabular}{lcccccccc}
\hline & \multicolumn{4}{c}{ Argila Montmorilonita } & \multicolumn{5}{c}{ Niobato Organofilizado } \\
\cline { 2 - 10 } & $\mathbf{O}_{2}$ & $\mathbf{d}\left(\mathbf{O}_{2}\right)$ & $\mathbf{H}_{2} \mathbf{O}$ & $\mathbf{d}\left(\mathbf{H}_{2} \mathbf{O}\right)$ & $\mathbf{O}_{2}$ & $\mathbf{d}\left(\mathbf{O}_{2}\right)$ & $\mathbf{H}_{2} \mathbf{O}$ & $\mathbf{d}\left(\mathbf{H}_{2} \mathbf{O}\right)$ \\
\hline $\mathbf{L L D P E}$ & 3,3 & 0,2 & 0,30 & 0,01 & 2,8 & 0,2 & 0,13 & 0,04 \\
$\mathbf{1 , 5 \%}$ & 3,2 & 0,1 & 0,23 & 0,02 & 2,5 & 0,2 & 0,11 & 0,02 \\
$\mathbf{2 , 5 \%}$ & 3,1 & 0,1 & 0,16 & 0,04 & 2,2 & 0,1 & 0,15 & 0,02 \\
$\mathbf{5 , 0 \%}$ & 2,9 & 0,1 & 0,17 & 0,04 & 2,7 & 0,1 & 0,15 & 0,02 \\
$\mathbf{7 , 5 \%}$ & 2,8 & 0,1 & 0,13 & 0,02 & 2,6 & 0,1 & 0,13 & 0,02 \\
$\mathbf{1 0 , 0 \%}$ & 2,6 & 0,2 & 0,18 & 0,01 & 2,3 & 0,1 & 0,13 & 0,01 \\
\hline
\end{tabular}

*Barrer $=\left[\left(10^{-11} \mathrm{~cm}^{3} \mathrm{O}_{2}\right) \cdot \mathrm{cm}\right] /\left[\mathrm{cm}^{2} . \mathrm{s} \cdot \mathrm{mmHg}\right]$. 
maneira de explicar essa seletividade ao vapor de água em relação ao oxigênio se deve a maior polaridade das moléculas de água, assim como a sua maior facilidade de condensação. Nesse caso nota-se que o efeito do volume do gás prevalece sobre o efeito da polaridade ao longo do processo de permeação de gás através dos nanocompósitos. Os valores de permeabilidade ao oxigênio obtidos neste trabalho estão consistentes com os encontrados na literatura ${ }^{[24,25]}$. O resultado do aumento da permeabilidade ao vapor de água para a amostra com $1,5 \%$ de argila, pode ser um indicativo de que nesta concentração, o compósito final apresenta uma maior quantidade de defeitos. Além disso, devido às diferenças na estrutura lamelar das nanocargas, os nanocompósitos com argila montmorilonita e os nanocompósitos com niobato organofilizado apresentaram propriedades distintas, como verificadas e discutidas anteriormente.

Em trabalhos que vem sendo desenvolvido em nosso laboratório, com este mesmo sistema ${ }^{[26]}$, porém enfocando especificamente aspectos relacionados com as correlações entre a morfologia e as propriedades de transporte, foi possível estimar a razão de aspecto (L/D) destes nanocompósitos. Isso é feito a partir de análises comparativas entre as imagens obtidas por microscopia eletrônica de transmissão (MET) e com base nos modelo de Nielsen e modificações sugeridas por Bharadwaj ${ }^{[27]}$. Neste caso a representação da curva com os valores experimentais de permeabilidade da matriz de LLDPE e dos compósitos em função da fração de carga inorgânica, foi comparada com diferentes curvas teóricas obtidas utilizando-se a equação do modelo de Nielsen, atribuindo-se diferentes valores de L/D. Os melhores resultados obtidos para ambos os sistemas, sem diferenças relevantes, situou-se na faixa compreendida entre $\mathrm{L} / \mathrm{D}=4$ a $\mathrm{L} / \mathrm{D}=10$, considerando-se o intervalo das concentrações de carga inorgânica entre $1,5 \%$ a $10,0 \%$ em massa. Teoricamente é conhecido que um tactóide totalmente esfoliado apresenta uma lamela com espessura da ordem de $1 \mathrm{~nm}$ (D) e comprimento da ordem de $100 \mathrm{~nm}$ (L), resultando numa razão de aspecto (L/D) da ordem de 100. Comparativamente, os resultados estimados, na faixa compreendida entre L/ $\mathrm{D}=4 \mathrm{a} / \mathrm{D}=10$, poderiam ser considerados extremamente baixos e pouco significativos do ponto de vista de seu efeito na tortuosidade inserida no material. Porém, a redução observada nos valores da permeabilidade ao vapor de água e ao oxigênio para os sistemas com argila montmorilonita Cloisite 20A e niobato organofilizado poderiam estar relacionados a dois possíveis fatores: a) ruptura dos tactoides, durante as etapas de processamento, levando a formação de empilhados de algumas lamelas com espessura superior a $1 \mathrm{~nm}$; b) boa distribuição e dispersão destes empilhados de lamelas na fase volume da matriz polimérica. Dessa forma, estes dois fatores parecem contribuir positivamente de forma sinérgica para o incremento da tortuosidade na matriz polimérica o que justificaria a redução observada nos valores de permeabilidade ao vapor de água e ao oxigênio, independentemente dos baixos valores estimados para a razão de aspecto.

\section{Conclusões}

A estrutura diferente das nanocargas influencia na propriedade mecânica e de barreira dos nanocompósitos. Isso pode ser visto pelos resultados do módulo elástico e da tenacidade, ou seja, os resultados foram superiores para os nanocompósitos preenchidos com argila montmorilonita do que preenchidos com niobato organofilizado. Portanto, de acordo com os resultados apresentados pode-se dizer que a interação entre o LLDPE/argila montmorilonita é mais intensa do que a interação observada entre o LLDPE/ niobato organofilizado. Os resultados de MEV mostraram que a distribuição da argila montmorilonita é superior àquela do niobato organofilizado para concentrações inferiores a $10,0 \%$.

Outro aspecto observado para o presente sistema nanométrico é que a polaridade dos gases é um fator que influencia na permeabilidade, ou seja, nesse caso quanto mais polar for o gás e maior for a sua capacidade de condensação, menor será o valor de permeabilidade. Além disso, observa-se que o processo de permeabilidade é mais sensível ao efeito da polaridade do gás do que ao volume das moléculas. Nesse caso ambos os nanocompósitos testados são mais seletivos ao vapor de água do que ao oxigênio.

Com base nos valores obtidos no ensaio de permeação, a nanocarga mais adequada a ser adicionada na matriz polimérica de LLDPE com o intuito de melhorar a propriedade de barreira de um filme para embalagem seria a argila montmorilonita com concentrações entre 2,5 a 10,0\% em massa como barreira ao vapor de água. Além disso, o niobato organofilizado, com concentração de até $3,0 \%$ em massa, seria o mais indicado para melhorar a barreira do filme de LLDPE ao oxigênio. Porém, como esses filmes precisam apresentar boa resistência à tração, então, seria necessário alcançar uma melhora nessa propriedade para os filmes com niobato organofilizado.

\section{Agradecimentos}

Os autores agradecem ao $\mathrm{CNPq}$ pelas bolsas de produtividade em pesquisa, Pos-doutoramento e doutoramento. E à FAPESP pelo apoio financeiro através do projeto Temático-2006/61008-5.

\section{Referências Bibliográficas}

1. Crippa, A.; Sydenstricker, T. H. D. \& Amico, S. C. - Polímeros, 17, p.188 (2007). http://dx.doi.org/10.1590/ S0104-14282007000300006

2. Teixeira, E. M. - "Correlação entre morfologia e propriedades de transporte de diclorometano no Poli(eter imida) (PEI) e na benda PET/PEI', Dissertação de Mestrado, Universidade Federal de São Carlos, Brasil (2002).

3. Bandi, S. \& Schiraldi, D. A. - Macromolecules, 39, p.6537 (2006). http://dx.doi.org/10.1021/ma0611826

4. Morales, A. R.; Cruz, C. V. M.; Peres, L. \& Ito, E. N. - Polímeros, 20, p.39 (2010). http://dx.doi.org/10.1590/ S0104-14282010005000004

5. Durmus, A.; Woo, M.; Kasgöz, A.; Macosko, C. W. \& Tsapatsis, M. - Eur. Polym. J., 43, p.3737 (2007). 
6. Golebiewski, J.; Rozanski, A.; Dzwonkowski, J. \& Galeski, A. - Eur. Polym. J., 44, p.270 (2008). http://dx.doi. org/10.1016/j.eurpolymj.2007.11.002

7. Ma, J.; Xu, J. ; Ren, J. H. ; Yu, Z. Z. \& Mai, Y. W. - Polymer, 44, p.4619 (2003). http://dx.doi.org/10.1016/ S0032-3861(03)00362-8

8. Brito, G. F.; Oliveira, A. D.; Araújo, E. M.; Melo, T. J. A.; Barbosa, R. \& Ito, E. N. - Polímeros, 18, p.170 (2008). http://dx.doi.org/10.1590/S0104-14282008000200015

9. Prado, L. A. S. A. \& Yoshida, I. V. P.- J. Non-Cryst. Solids, 355, p.1726 (2009).

10. Xu, B.; Zheng, Q.; Song, Y. \& Shangguan, Y. - Polymer, 47, p.2904 (2006). http://dx.doi.org/10.1016/j. polymer.2006.02.069

11. Villanueva, M. P.; Cabedo, L.; Lagarón, J. M. \& Gimenez, E. - J. Appl. Polym. Sci., 115, p.1325 (2010). http://dx.doi. org/10.1002/app.30278

12. Gasperin, M. \& Bihan, M. T. J. - Solid State Chem., 43, p.346 (1982). http://dx.doi.org/10.1016/0022-4596(82)90251-1

13. Ray, S. S. \& Okamoto, M. - Prog. Polym. Sci., 28, p. 1539 (2003).

14. Bizeto, M. A. \& Constantino, V. R. L. - Mater. Res. Bull., 39, p.1729 (2004). http://dx.doi.org/10.1016/j. materresbull.2004.05.001

15. Chausson, S.; Caignaert, V.; Retoux, R.; Rueff, J. M.; Pluart, L. L.; Madex, P. J. \& Jafrés, P. A. - Polymer, 49, p.488 (2008). http://dx.doi.org/10.1016/j.polymer.2007.11.050

16. Pavlidou, S. \& Papaspyrides, C. D. - Prog. Polym. Sci., 33, p.1119 (2008). http://dx.doi.org/10.1016/j. progpolymsci.2008.07.008
17. Shiguihara, A. L. - "Investigação dos processos de intercalação e esfoliação de hexaniobato lamelar $e$ preparação de materiais híbridos com biopolímeros", Tese de Doutorado, Universidade de São Paulo, Brasil (2010).

18. Alonso, J. M. H.; Sedlakova, Z. \& Marand, E. - J. Membr. Sci., 349, p.251 (2010). http://dx.doi.org/10.1016/j. memsci.2009.11.057

19. Choudalakis, G. \& Gotsis, A. D. - Eur. Polym. J., 45, p.967 (2009). http://dx.doi.org/10.1016/j.eurpolymj.2009.01.027

20. Otaguro, H. \& Rúvolo-Filho, A. C. - Int. Polym. Proc., 4, p.406 (2012). http://dx.doi.org/10.3139/217.2384

21. Formes, T. D. \& Paul, D. R. - Polymer, 44, p.4993 (2003). http://dx.doi.org/10.1016/S0032-3861(03)00471-3

22. Duncan, T. V. - J. Colloid Interface Sci., 363, p.1 (2011).

23. Paul, D. R. \& Robeson, R. L. - Polymer, 49, p.3187 (2008). http://dx.doi.org/10.1016/j.polymer.2008.04.017

24. Hotta, S. \& Paul, D. R. - Polymer, 45, p.7639 (2004). http:// dx.doi.org/10.1016/j.polymer.2004.08.059

25. Villaluenga J. P. G. \& Seoane, B. - Polymer, 39, p.3955 (1998). http://dx.doi.org/10.1016/S0032-3861(98)00005-6

26. Harumi Otaguro. - "Correlações entre o processamento e propriedades mecânicas, térmicas e de transporte de gases, vapor orgânico e água em nanocompósitos de matriz de polietileno linear de baixa densidade", CNPq-Pósdoutorado-Processo número: 15735/2009-0, Universidade Federal de São Carlos, Brasil, (2009-2010).

27. Bharadwaj, R. K. - Macromolecules, 34, p.9189 (2001). http://dx.doi.org/10.1021/ma010780b

Enviado: $24 / 04 / 12$ Reenviado: $12 / 12 / 12$ Aceito: $15 / 01 / 13$ 